\title{
Calibração de sondas capacitivas, funções físico-hídricas e variação do armazenamento de água em um argissolo cultivado com palma forrageira ${ }^{1}$
}

\author{
Jorge Torres de Araújo Primo², Thieres George Freire da Silva*3, Sérvulo Mercier Siqueira e Silva \\ Magna Soelma Beserra de Moura ${ }^{5}$, Luciana Sandra Bastos de Souza ${ }^{6}$
}

http://dx.doi.org/10.1590/0034-737X201562010003

\begin{abstract}
RESUMO
Objetivou-se, com este trabalho, realizar a calibração de três sondas capacitivas, visando a utilizá-las na definição de funções físico-hídricas e na análise da variação espacial e temporal do conteúdo $(\theta)$ e do armazenamento de água, em um Argissolo Vermelho-Amarelo, cultivado com três clones de palma forrageira, no Semiárido pernambucano. O estudo foi executado numa área experimental situada em Serra Talhada, PE. Para isso, foi realizada a calibração de três sondas capacitivas, usando-se dados de densidade aparente do solo, de umidade gravimétrica e de frequências relativas, em cinco condições de umidade do solo. Aplicou-se o método do perfil instantâneo, para determinação do fluxo vertical de água, e do potencial matricial de água no solo. Em seguida, analisou-se a variação espaço-temporal do $\theta$ e do armazenamento de água no solo. As medidas de frequência relativa das sondas capacitivas apresentaram elevada relação com os dados de $\theta\left(\mathrm{R}^{2}>0,87\right)$. A partir do método do perfil instantâneo, foi possível determinar as funções da condutividade hidráulica e do potencial total de água no solo, em relação ao $\theta$, com elevados coeficientes de determinação $\left(\mathrm{R}^{2}>0,91\right)$. Não houve diferenças significativas do $\theta$ entre os clones de palma forrageira.
\end{abstract}

Palavras-chave: Diviner, método do perfil instantâneo, potencial total de água no solo.

\section{ABSTRACT}

\section{Field calibration of capacitive probes, physical-hydric propriety and variation of water storage in soil cultivated with cactus forage}

The objective of this work was to carry out calibration of three capacitive probes, to use them in the definition of physical-hydric functions and in spatial and temporal analysis of the soil water content (è) and water storage in an Udult soil cultivated with three palm forage clones, in Brazilian Semi-arid. The study was carried out in an experimental area located in Serra Talhada, State of Pernambuco. Therefore, calibration of the three capacitive probes was carried out using soil density and gravimetric moisture data and relative frequencies in five conditions of soil moisture. It was applied the instantaneous profile method for determination of water flow in the soil and matrix potential of the water in the soil. After that, the space-temporal variation of è and water storage in the soil were

\footnotetext{
Submetido em 08/05/2013 e aprovado em 17/09/2014.

${ }^{1}$ Parte da dissertação de mestrado do primeiro autor, apresentada ao Programa de Pós-graduação em Produção Vegetal, Universidade Federal Rural de Pernambuco/Unidade Acadêmica de Serra Talhada, financiada pela FACEPE / CNPq / CAPES / UFRPE.

${ }^{2}$ Universidade Federal Rural de Pernambuco, Unidade Acadêmica de Serra Talhada, Serra Talhada, Pernambuco, Brasil. jorgetaprimo@ hotmail.com

${ }^{3}$ Universidade Federal Rural de Pernambuco, Unidade Acadêmica de Serra Talhada, Serra Talhada, Pernambuco, Brasil. thieres_freire@ yahoo.com.br

${ }^{4}$ Instituto Agronômico de Pernambuco, Serra Talhada, Pernambuco, Brasil. servulo.siqueira @ipa.br

${ }^{5}$ Empresa Brasileira de Pesquisa Agropecuária, Embrapa Semiárido, BR 428, Km 152, Zona Rural, Caixa Postal 23, 56310-000, Petrolina, Pernambuco, Brasil. magna @ cpatsa.embrapa.br

${ }^{6}$ Universidade Federal Rural de Pernambuco, Unidade Acadêmica de Serra Talhada, Serra Talhada, Pernambuco, Brasil. sanddrabastos@yahoo.com.br

"Autor para correspondência: thieres_freire@yahoo.com.br
} 
analyzed. Measures of relative frequency of the capacitive probes showed high relationship with the è data $\left(\mathrm{R}^{2}>\right.$ 0.87). From the instantaneous profile method, it was possible to determine the functions of soil hydraulic conductivity and soil water total potential, both in terms of è, with high coefficients of determination $\left(R^{2}>0.91\right)$. There were no significant differences of the è between palm forage clones.

Key words: Diviner probe, instantaneous profile method, soil water total potential.

\section{INTRODUÇÃO}

Por ser, tradicionalmente, uma cultura semiperene, de colheita bienal, a palma forrageira pode, ao longo do seu ciclo produtivo, sofrer modificações no crescimento e no desenvolvimento, em resposta às alterações sazonais e interanuais das condições do ambiente (Dubeux Júnior et al., 2006; Oliveira, 2008). Essas condições, atreladas às características edáficas, conferem uma ampla variação do armazenamento de água no solo $(\Delta \mathrm{A})$, que pode refletir a interação do ambiente com a cultura. É um dado que condiciona o conteúdo de água no solo $(\theta)$ e que sofrerá influência de aspectos relacionados com a topografia, propriedades físicas do solo, cobertura vegetal e condições meteorológicas locais. Por sua vez, $\theta$ é uma informação bastante utilizada em estudos que envolvem a infiltração de água no solo, estimativa da condutividade hidráulica, drenagem, irrigação, evapotranspiração, entre outros (Rossato et al., 2004; Santos et al., 2011).

Assim, para a análise da $\Delta \mathrm{A}$, é necessário o monitoramento do conteúdo de água do solo, que pode ser obtido por meio de métodos diretos e indiretos. A gravimetria é considerada o método direto padrão, enquanto os métodos indiretos são aqueles que dependem de propriedades físicas associadas à solução dos solos, para se determinar o conteúdo de água do solo. São exemplos desses tipos de métodos que se baseiam na reflectometria no domínio do tempo (TDR), a contabilização da passagem de nêutrons, a resistência elétrica e a frequência no domínio do tempo (FDR) (Andrade Junior et al., 2007).

A FDR é denominada, comercialmente, 'sonda capacitiva’, por exemplo, a Diviner@2000 (Sentek Pty Ltd., Australia). Embora os fornecedores disponibilizem uma equação universal de estimativa do conteúdo de água no solo em função da frequência relativa obtida pelo sensor, a calibração local pode melhorar a precisão das medições (Silva et al., 2008). As sondas Diviner@2000 são citadas em estudos de dinâmica de água no solo (Martín-Vertedor et al., 2011; Zhang et al., 2010).

Nesse tipo de estudo, $\theta$ também é utilizada em uma das etapas mais importantes, que se trata da estimativa do fluxo vertical de água no solo (Q). Q depende da de- terminação da condutividade de água do solo, numa condição de solo não saturado, $K(\theta)$. Para isso, tem-se o método do perfil instantâneo, que apresenta a vantagem de estabelecer a relação da $\mathrm{K}$ em função de $\theta$, utilizando-se medidas simultâneas de potencial matricial e de $\theta$, em campo, em diferentes tempos e profundidades. Esse método tem sido aplicado com muito sucesso em vários estudos (Ghiberto et al., 2011; Martins, 2009; Silva, 2005). No cálculo de Q, prevê-se também o uso do potencial total de água no solo $(\varnothing \mathrm{t})$, para determinação do gradiente $(\Delta \psi \mathrm{t} / \Delta \mathrm{z})$. Em campo, $\psi \mathrm{t}$ comumente é determinado mediante medidas tensiométricas e valores de $\mathrm{z}$. Todavia, naqueles casos em que se usam equipamentos de medida indireta do conteúdo de água do solo (sonda de nêutrons, Diviner@2000), భt pode ser obtido por curvas semiempíricas em função de $\theta$, dentre elas o modelo Exponencial (Dourado-Neto et al., 2000).

Com base no exposto, objetivou-se, com este trabalho, realizar a calibração de três sondas capacitivas, visando a utilizá-las na definição de funções físico-hídricas e na análise da variação espacial e temporal do conteúdo de água do solo e do armazenamento de água, em um Argissolo Vermelho-Amarelo, cultivado com três clones de palma forrageira, em condições de sequeiro, no Semiárido pernambucano.

\section{MATERIAL E MÉTODOS}

\section{Características da área experimental e delineamento experimental}

$\mathrm{O}$ estudo foi conduzido em uma área de sete hectares, de cultivos experimentais de palma, pertencente ao Instituto Agronômico de Pernambuco - IPA (município de Serra Talhada, 759'S, 38¹5' O e 461 m, no Semiárido do Estado de Pernambuco), que apresenta solo do tipo Argissolo Vermelho-Amarelo eutrófico franco arenoso, conforme a classificação da Embrapa (1999). Dentro dessa área, foram delimitadas duas subáreas representativas, entre os experimentos de palma, uma para a calibração de três sondas capacitivas e outra para aplicação do método do perfil instantâneo e, por conseguinte, definição de funções físico-hídricas do solo. 
Além disso, selecionou-se, nas proximidades das duas subáreas representativas, um dos experimentos com plantio de três clones de palma forrageira, iniciado em fevereiro de 2010 e conduzido em condições de sequeiro, visando à análise da variação espacial e temporal do conteúdo e do armazenamento de água no solo. Nessa análise, os tratamentos foram representados pelos clones IPA-Sertânia (IPA-200205), do gênero Nopalea, Miúda (IPA-100004), do gênero Nopalea, e Orelha de Elefante Mexicana (IPA-200016), do gênero Opuntia, distribuídos em três blocos ao acaso, subdivididos em parcelas experimentais aleatórias. As parcelas foram arranjadas em quatro fileiras, com 20 plantas cada uma, ocupando área de 25,6 m² e área útil de $10,24 \mathrm{~m}^{2}$. As fileiras de cultivo foram espaçadas em 1,6 m x 0,2 m, em curvas de nível, compreendendo uma densidade equivalente de 31.250 plantas por hectare. Foram realizadas capinas manuais, aplicação de herbicida e de inseticidas quando necessário para manutenção da área livre de plantas espontâneas e de pragas, respectivamente.

\section{Monitoramento da frequência relativa ao longo do ciclo dos clones de palma forrageira e calibração de sondas capacitivas}

O monitoramento da frequência relativa (FR) foi realizado ao longo do tempo, na área de cultivo dos clones de palma forrageira, usando-se uma das sondas capacitivas, dentre as três calibradas neste estudo, como será descrito em seguida. Esses dados de FR foram, posteriormente, utilizados na estimativa do conteúdo $(\theta)$ e do armazenamento de água no solo. Para isso, entre os meses de setembro e outubro de 2010 (sétimo e oitavo meses após o plantio) foram instalados nove tubos de acesso, conforme instruções do fabricante, um em cada repetição dos clones, a uma distância de $0,10 \mathrm{~m}$ das fileiras de cultivo. O monitoramento da FR foi feito a cada $0,10 \mathrm{~m}$, em intervalos de tempo de três dias, entre $20 \mathrm{de}$ outubro de 2011 e 02 de março de 2012, totalizando 499 dias.

A calibração de três sondas capacitivas (modelo Diviner@2000 Sentek Pty Ltda., Austrália) foi realizada apenas entre os meses de setembro e outubro de 2012 (décimo nono e vigésimo meses após o plantio). Para isso, foram instalados tubos de acesso, adjacentes à área experimental. As calibrações foram feitas por meio da adaptação de método citado pelo fabricante (Sentek, 2000). Assim, além de estabelecer três níveis de umidade do solo (seco, úmido e saturado), foram adotados mais dois níveis, semisseco e subúmido, representando o conteúdo de água do solo superior ao da condição seca e inferior ao da condição úmida, respectivamente, visando a contemplar toda a faixa de conteúdo de água do solo. Dois tubos foram considerados para cada condição "seca", "úmida" e "saturada" e um para cada condição "semisseca" e "subúmida", totalizando oito tubos de acessos. Após 25 dias da instalação dos tubos de acesso foram abertas, manualmente, três trincheiras, com dimensões de 4,0 m x 1,5 m x 1,0 m de profundidade, com o intuito de realizar os procedimentos de calibração das sondas capacitivas. Uma das trincheiras foi usada para dois tubos da condição "seca" e o da condição "semisseca", uma para os dois tubos da condição "úmida" e o da condição "subúmida", e outra para os dois tubos da condição "saturada". A condição seca foi estabelecida no mês de setembro de 2012, quando os níveis pluviométricos foram praticamente nulos $(1,4 \mathrm{~mm})$, e nos meses antecedentes (abril a agosto de 2012) totalizaram apenas $35,60 \mathrm{~mm}$ e a demanda atmosférica foi de $783 \mathrm{~mm}$.

Por meio de um anel de infiltração com diâmetro de $0,50 \mathrm{~m}$ e $0,50 \mathrm{~m}$ de altura, com exceção dos dois tubos para a condição seca, os demais receberam 460 L de água até a frente de molhamento, observada ao longo da trincheira, atingir 1,0 metro de profundidade. No dia seguinte à aplicação de água, foram feitas as leituras com a sonda e a coleta das amostras de solo junto aos tubos da condição "saturada". Por sua vez, para os tubos do nível úmido, as leituras e amostragens foram realizadas sete dias após a colocação da água no solo. Para a condição "subúmida", foi necessário esperar 18 dias, enquanto, para a condição "semisseca", 28 dias, por causa do avanço lento da frente de umedecimento do solo. Como os procedimentos de calibração foram feitos no período seco do ano, para os dois tubos do nível "seco" procedeu-se apenas às leituras e coleta do solo.

Em cada tubo de acesso, foram realizadas três leituras de frequência relativa (FR), gerando-se uma média para cada uma das camadas (a cada 0,10 m, de 0,10 a 0,70 m). Em seguida, procedeu-se à coleta do solo para as determinações da umidade gravimétrica e da densidade aparente do solo. Para isso, uma amostra de solo deformada e duas indeformadas (anel volumétrico 55 x $45 \mathrm{~mm}$ ) foram retiradas em áreas adjacentes ao tubo e colocadas em uma lata de alumínio ( 81 x $75 \mathrm{~mm}$ ) com tampa. As amostras foram pesadas, secadas em estufa $\left(105^{\circ} \mathrm{C}\right)$ e pesadas, novamente, após $48 \mathrm{~h}$. O conteúdo de água do solo $\left(\theta, \mathrm{m}^{3} \mathrm{~m}^{-3}\right)$, em cada camada, foi determinado, multiplicando-se a umidade gravimétrica pela densidade do solo $\left(\mathrm{kg} \mathrm{m}^{-3}\right)$. Os valores de $\theta$ e as correspondentes leituras de FR foram submetidos à análise de regressão $\left(\theta=\mathrm{a} \cdot \mathrm{FR}^{\mathrm{b}}+\mathrm{c}\right)$ e a sua significância e de seus parâmetros foi avaliada mediante teste F e t de Student, respectivamente, a 5\% de significância. 


\section{Definição de funções físico-hídricas do solo}

As funções físico-hídricas do solo foram estabelecidas para a condutividade hidráulica do solo não saturado e para a estimativa do potencial total de água do solo, ambos ao longo do perfil do solo, assumindose como variável independente o conteúdo de água do solo. Para isso, aplicou-se, em campo, entre os meses de novembro e dezembro de 2012, o método do perfil instantâneo, para a determinação dos componentes da equação de Richard, o que melhor representa as condições reais das propriedades físico-hídricas do solo, conforme Libardi (2005). Para aplicação do método do perfil instantâneo, foi preparada uma área plana, adjacente às parcelas experimentais, onde se inseriu uma estrutura de chapa galvanizada ( $2 \mathrm{~mm}$ de espessura), em formato retangular, com 1,0 m de altura, 2,0 m de largura e 2,0 m de comprimento, a uma profundidade de $0,7 \mathrm{~m}$, no solo, para evitar fluxo lateral, no período de inundação e de redistribuição de água. Dentro da área $\operatorname{dos} 4,0 \mathrm{~m}^{2}$, foram instalados oito tensiômetros, nas profundidades de 0,10 a $0,80 \mathrm{~m}$, a cada $0,10 \mathrm{~m}$ de intervalo, com o recipiente de mercúrio posicionado a 0,36 $\mathrm{m}$ da superfície do solo, bem como um tubo de acesso para monitoramento simultâneo do conteúdo de água no solo, por meio das três sondas capacitivas calibradas, conforme descrito anteriormente. A saturação do perfil foi iniciada, colocando-se água na área dos 4,0 $\mathrm{m}^{2}$, por meio de uma tubulação de "PVC", oriunda do prolongamento do sistema de irrigação da área experimental, perdurando até o momento em que se verificou pouca variação das leituras dos tensiômetros ao longo do perfil. A partir da interrupção do fornecimento de água, a área dos $4 \mathrm{~m}^{2}$ foi coberta com uma lona plástica lisa de cor preta, para evitar o fluxo vertical da água no solo, exercido pelo efeito da evaporação. As medidas dos tensiômetros, ao longo do tempo de redistribuição de água no solo, foram utilizadas para o cálculo do potencial total do solo $(\psi t)$.

Assim, por meio de medidas simultâneas do conteúdo de água do solo e do potencial total de água medido durante o período de redistribuição (699 horas), foi possível calcular a densidade de fluxo e o gradiente de potencial total, de modo a se obter a condutividade hidráulica e, por conseguinte, a relação com o conteúdo de água do solo. Para isso, inicialmente, encontrou-se a solução da equação de Richards. Para o cálculo da condutividade hidráulica do solo (K), foram resolvidos os problemas de contorno dessa equação, conforme descrito por Libardi (2005), e os resultados foram relacionados com os dados de profundidade (em metros) de cada camada $\mathrm{L}$. Com os valores de $\mathrm{A}_{\mathrm{L}}$, t, $\psi \mathrm{t}$ e $\mathrm{z}$, foram escolhidos os melhores ajustes matemáticos a partir de equações quadráticas, de modo a se obter os gradientes da equação resolvida de Richards. Posteriormente, com os valores de $\mathrm{K}$ e $\psi \mathrm{t}$, resultantes do método do perfil instantâneo, foram relacionados com as medidas do conteúdo de água do solo, conforme Libardi (2005) e Dourado-Neto et al. (2000), sendo avaliado o desempenho dos ajustes por meio dos valores dos coeficientes de determinação.

\section{Armazenamento de água no solo cultivado com três clones de palma}

$\mathrm{O}$ armazenamento de água no solo (A) foi obtido, para intervalos de 14 dias, por meio da integração dos valores de $\theta$ de todas as profundidades. Os resultados foram integralizados para nove períodos representativos do ciclo da cultura, visando à melhor interpretação dos dados. Tanto os dados de $\theta$ quanto os de A para os clones de palma forrageira foram comparados entre si por meio de teste t de Student, a 5\% de significância.

\section{Monitoramento da precipitação pluviométrica}

Os dados de precipitação foram obtidos numa PCD (plataforma de coleta de dados automatizada), pertencente à Agência Pernambucana de Águas e Clima - APAC/ ITEP, localizada, aproximadamente, a $700 \mathrm{~m}$ da área, e obtidos no site do Centro de Previsão do Tempo e Estudos Climáticos-CPETC/INPE (www.cptec.inpe.br).

\section{RESULTADOS E DISCUSSÃO}

\section{Calibração das sondas capacitivas}

As leituras médias de conteúdo de água do solo $(\theta)$ e de frequência relativa (FR), das três sondas capacitivas, para cada profundidade, nas cinco condições de umidade propostas (saturada, úmida, subúmida, semisseca e seca), são mostradas na Figura 1. Os valores de $\theta$ variaram de $0,0621 \mathrm{~m}^{3} \mathrm{~m}^{-3}$, na profundidade de $0,10 \mathrm{~m}$, na condição seca, até $0,3236 \mathrm{~m}^{3} \mathrm{~m}^{-3}$, na profundidade de $0,60 \mathrm{~m}$, na condição saturada, associados a valores de FR de 0,4744 $\pm 0,0146$ e $0,8166 \pm 0,0161$, nessa ordem.

Os valores de $\theta$ ao longo do perfil obedeceram às suas respectivas condições impostas de umedecimento do solo, mas, de modo geral as maiores umidades foram constatadas com a profundidade (>0,40 m), em resposta ao avanço da frente de molhamento. Nas condições "úmida" e "subúmida", mesmo as amostras sendo obtidas em dias distintos (sete e 18 dias após a plena infiltração dos 460 L de água, os valores de umidade estiveram bastante próximos, diferindo apenas nas primeiras camadas do solo, que apresentam maior interação com a atmosfera e menor condutividade hidráulica do solo, que tende a diminuir com a profundidade.

A tendência dos valores de FR obedeceu àqueles de $\theta$ (Figura 1B). Percebe-se que as leituras de FR entre as 
sondas capacitivas apresentaram pequeno desvio entre si, confirmando as informações, citadas pelo fabricante, de que a normalização das leituras de frequência permite obter maior exatidão nas leituras de $\theta$, uma vez que diferentes sondas tendem realizar leituras distintas em condições específicas como o ar e a água. As densidades aparentes do solo foram iguais a 1,71, 1,66, 1,64, 1,66 e $1,62 \mathrm{~kg} \mathrm{~m}^{-3}$, para os cinco níveis de umedecimento do solo (saturada, úmida, subúmida, semisseca e seca), respectivamente, resultando numa média de 1,66 $\pm 0,06$ $\mathrm{kg} \mathrm{m}^{-3}$. Essas diferenças podem estar relacionadas com as dificuldades de amostragens indeformadas do solo, logo que a presença de cascalhos é preponderante ao longo do perfil. Na Figura 1B, é possível verificar que os maiores valores de densidade do solo estão ligeiramente situados nas primeiras camadas, onde a presença de argila é menor do que à profundidade de $0,40 \mathrm{~m}$.

Na Figura 2, constata-se que os dados da calibração para as três sondas capacitivas ficaram bem próximos entre si, estando sua dispersão em relação à curva associada, sobretudo, à variabilidade dos valores de densidade apa- rente do solo, conforme relatado por Silva et al. (2008). Os coeficientes de determinação obtidos para todo o perfil com as três sondas foram elevados $\left(R^{2}>0,87\right)$ (Tabela 1), estando dentro da faixa observada em vários estudos, sobretudo para aqueles solos que apresentam maiores densidades, como os obtidos por Fares et al. (2004) e Silva et al. (2008).

Comparando-se os resultados, obtidos neste estudo, com a calibração do fabricante e de outros experimentos de campo, encontrados na literatura (Tabela 1), as curvas de calibração das três sondas capacitivas ficaram próximas daquela recomendada pelo fabricante, sendo o desvio médio de $6,-1$ e $-7 \%$, nessa ordem, para as sondas N1, N2 e N3. Andrade Junior et al. (2007) citam que essas diferenças estão associadas ao tipo, à granulometria e à estrutura do solo. A equação proposta por esses autores também apresentou boa proximidade, sendo a diferença, em média, de apenas $-3 \%$. Esses resultados devem-se ao fato de o fabricante e Andrade Júnior et al. (2007) terem feito as suas calibrações em solos com textura arenosa, como a verificada neste estudo. A

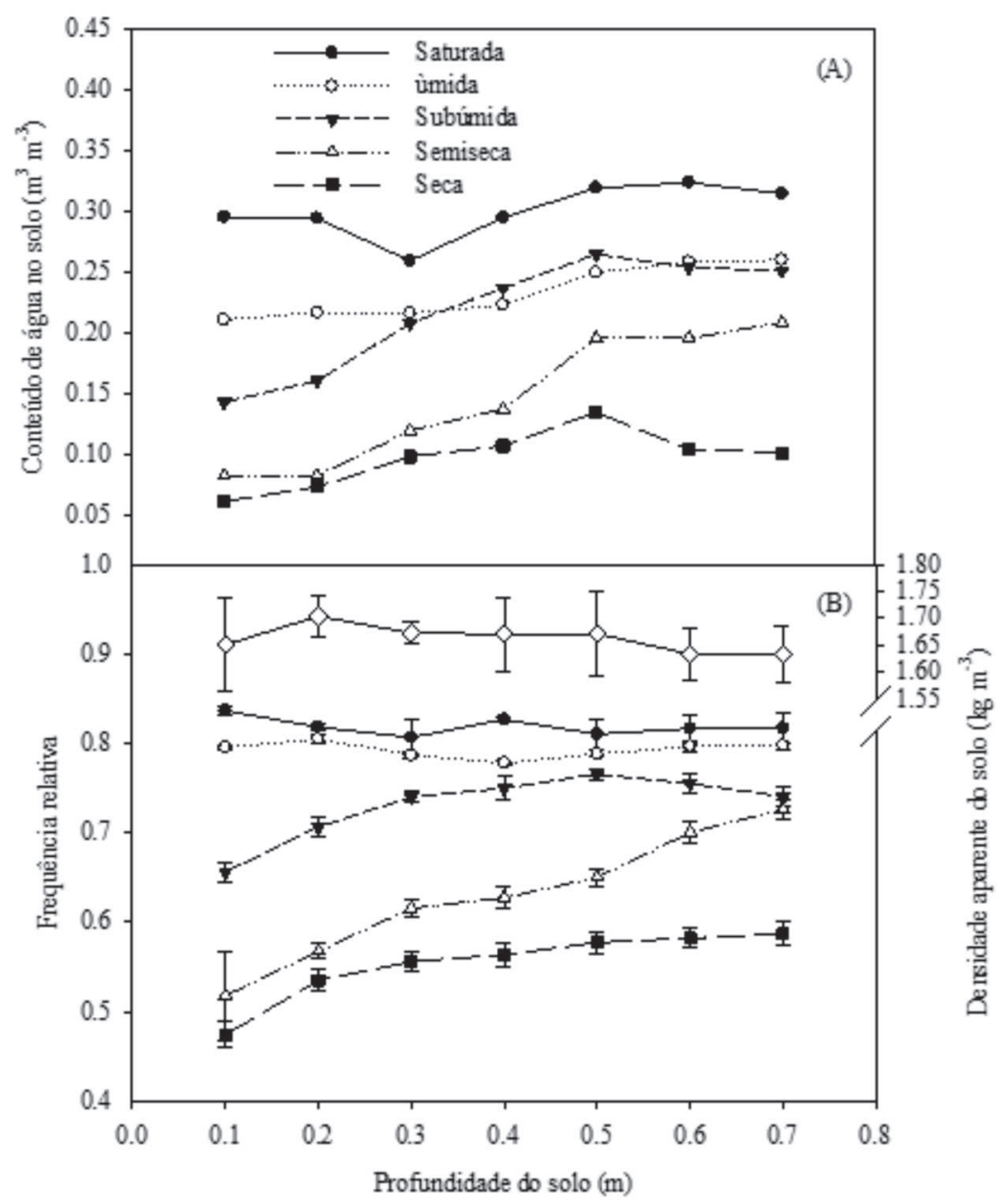

Figura 1. Conteúdo de água no solo (A), e frequência relativa e densidade aparente do solo (B) em cinco condições de umidade (saturada, úmida, subúmida, semisseca e seca) ao longo do perfil (0,1 - 0,7 m) num Argissolo Vermelho-Amarelo cultivado com clones de palma forrageira, em Serra Talhada, Semiárido pernambucano. 
calibração de Groves \& Rose (2004) apresentou desvio semelhante ao da Sonda N2, apesar de o experimento ter sido conduzido em solo com textura bem distinta da do solo deste estudo (Tabela 2). Por sua vez, a calibração de Silva et al. (2008) apresentou diferença de $10 \%$, em relação à do fabricante. Os maiores desvios verificados em relação à calibração do fabricante foram para as calibrações propostas por Fares et al. (2004), Hidalgo et al. (2003) e Paltineanu \& Starr (1997), em torno de 32,36 e $39 \%$, respectivamente.

\section{Características físico-hídricas do solo}

Com a aplicação do método do perfil instantâneo, foram obtidos os coeficientes das equações de ajuste da condutividade hidráulica do solo $(K(\theta))$ e do potencial total de água $(\psi t)$ no solo, ambos em função do $\theta$, para cada uma das camadas do perfil do solo em estudo, conforme mostrado na Tabela 2, respectivamente. As equações de $\mathrm{K}(\boldsymbol{\theta})$ apresentaram ótimos desempenhos, com valores de coeficiente de determinação elevados (>0,9619), sendo os melhores ajustes para as camadas mais superiores. Ótimos desempenhos também foram encontrados por Martins (2009) ( $\mathrm{R}^{2}$ entre 0,95 e 0,98), Carvalho et al. (2007) ( $\mathrm{R}^{2}$ entre 0,977 e 0,999), Jong van Lier \&Libardi (1999) $\left(\mathrm{R}^{2}\right.$ entre 0,786 e 0,999$)$ e Gonçalves (2011) $\left(\mathrm{R}^{2}\right.$ $>0,973)$, em condições edafoclimáticas distintas entre si e também em relação à deste estudo.

Os valores dos parâmetros Ko e $\gamma$ apresentaram uma grande variação (C.V. de 75,94 e 36,94\%), confirmando a alta variabilidade do perfil do solo, como encontrada por Jong van Lier \& Libardi (1999), indicando que o uso de valores médios pode provocar erros consideráveis na es-

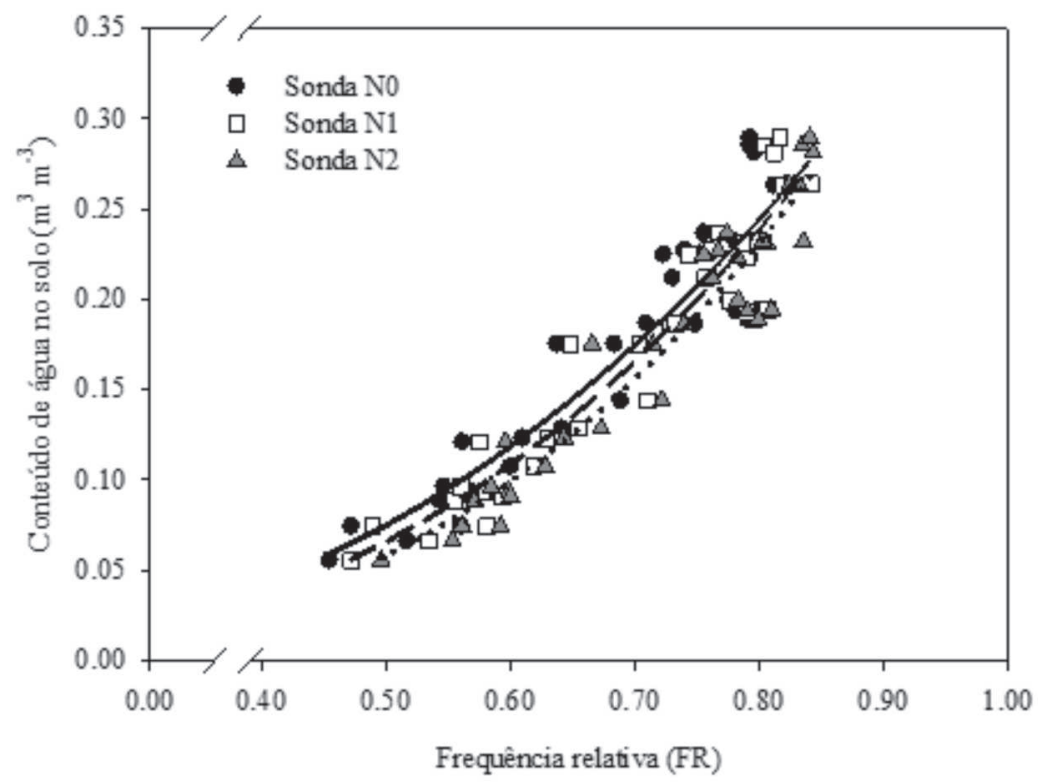

Figura 2. Curvas de calibração de três sondas capacitivas (Diviner@ 2000) para um Argissolo Vermelho-Amarelo cultivado com clones de palma forrageira, em Serra Talhada, Semiárido pernambucano.

Tabela 1. Calibração de sondas capacitivas utilizadas para o monitoramento do conteúdo de água no solo da área experimental em comparação àquelas relatadas na literatura

\begin{tabular}{|c|c|c|c|c|c|c|}
\hline \multirow{2}{*}{ Fonte } & \multicolumn{5}{|c|}{ Granulometria (g/kg1) da Equação } & \multirow{2}{*}{$\mathbf{R}^{2}$} \\
\hline & Areia & Silte & Argila & $\mathrm{kg} \mathrm{m}^{-3}$ & & \\
\hline Sonda NO & 684 & 253 & 64 & 1,66 & $\boldsymbol{\theta}_{\mathrm{v}}=0,4279 . \mathrm{FR}^{2,5116}$ & 0,87 \\
\hline Sonda N1 & 684 & 253 & 64 & 1,66 & $\boldsymbol{\theta}_{\mathrm{v}}=0,4395 . \mathrm{FR}^{2,7350}$ & 0,89 \\
\hline Sonda N2 & 684 & 253 & 64 & 1,66 & $\boldsymbol{\theta}_{\mathrm{v}}^{v}=0,4453 \cdot \mathrm{FR}^{2,9403}$ & 0,92 \\
\hline Fabricante & - & - & - & - & $\boldsymbol{\theta}_{\mathrm{v}}=0,494 . \mathrm{FR}^{3,017}$ & 0,99 \\
\hline Groves e Rose (2004) & 280 & 280 & 440 & 1,09 & $\boldsymbol{\theta}_{\mathrm{v}}=0,514 \cdot \mathrm{FR}^{3,371}$ & 0,93 \\
\hline Fares et al. (2004) & 394 & 122 & 484 & 1,52 & $\boldsymbol{\theta}_{\mathrm{v}}=0,440 . \mathrm{FR}^{1,549}-0,040$ & 0,88 \\
\hline Hidalgo et al.(2003) & 70 & 410 & 520 & 1,28 & $\boldsymbol{\theta}_{v}=0,382 \cdot \mathrm{FR}^{1,616}$ & 0,81 \\
\hline Andrade Júnior et al. (2007) & 846 & 58 & 95 & 1,45 & $\boldsymbol{\theta}_{v}=0,397 . \mathrm{FR}^{2,533}$ & 0,97 \\
\hline Silva et al. (2008) & 669 & 100 & 231 & 1,59 & $\boldsymbol{\theta}_{\mathrm{v}}=0,492 . \mathrm{FR}^{2,757}$ & 0,93 \\
\hline Paltineanu \& Starr (1997) & 350 & 560 & 90 & 1,41 & $\boldsymbol{\theta}_{\mathrm{v}}=0,490 . \mathrm{FR}^{2,167}$ & 0,99 \\
\hline
\end{tabular}

$\theta=$ conteúdo de água no solo, $\mathrm{FR}=$ frequência relativa. 
timativa do fluxo de água desse solo. Observa-se uma tendência de redução da condutividade hidráulica do solo saturado com a profundidade. Resultados similares foram citados por Silva et al. (2012), para dois Argissolos da Bacia do Jatobá e de Mimoso, no município de Pesqueira, PE, em ambiente do Semiárido brasileiro, quando os valores variaram de 0,0014 a $0,00024 \mathrm{~m} \mathrm{~h}^{-1}$, entre as profundidades de 0,15 a $0,55 \mathrm{~m}$. Os valores de $\gamma$ variaram entre 30 e 100, com os maiores valores aumentando com a profundidade. Esses resultados são próximos aos encontrados por Martins (2009), variando de 40 a 100, para solos do tipo Latossolo Amarelo e Nitossolo Vermelho, com textura arenosa e argilosa, densidade médias de 1,61 e $1,30 \mathrm{~kg} \mathrm{~m}^{-3}$ e porosidade total de 39,5 e $54,5 \%$, nessa ordem. Carvalho et al. (2007) também obtiveram valores de $\gamma$ entre 41,49 e 101,68.

Porém, Jong van Lier \& Libardi (1999) indicam que esses valores podem superar 500. O aumento dos valores de $\gamma$ pode estar associado ao aumento do teor de argila do solo. Essa relação também foi encontrada por Prevedello et al. (1981), que verificaram diminuição dos seus valores com a redução do teor de argila, em solos de distintas texturas. Diferentemente dos valores de Ko e $\gamma$, a $\theta$ o, equivalente ao conteúdo de água no momento da saturação do solo, não apresentou muita variação no perfil do solo (C.V. $=7,27 \%)$, entretanto, os maiores valores de $\theta$ o são das profundidades maiores, o que pode estar associado ao maior teor de argila nessas camadas.

Semelhantemente aos ajustes da $K(\theta)$, as equações do potencial total de água $(\psi \mathrm{t})$ do solo apresentaram valores de coeficiente de determinação elevados $\left(\mathrm{R}^{2}>\right.$ 0,9159) (Tabela 2). Os melhores resultados foram observados para as camadas mais superficiais, onde os valores de á foram os menores, indicando a ocorrência dos $\psi \mathrm{t}$ maiores, ou seja, menos negativos, e os seus valores variam menos com o $\theta$, quando comparados com os das maiores profundidades, logo que os valores de $\beta$ são menores. A variação dos valores de á é elevada entre as camadas do solo (118,69\%), demonstrando a estratificação das características ao longo do perfil, semelhante àquela verificada para a condutividade hidráulica do solo. Uma menor variação foi verificada, em relação dos valores de $\beta$, quanto à resposta do $\psi$ t ao è. Com isso, a partir dos dados ajustados de $\alpha$ e $\psi$ t será possível a estimativa do potencial total de água no solo, na ausência de medidas tensiométricas e com o uso de sensores eletrônicos que fornecem leituras de $\theta$, a serem aplicados em estudos de balanço de água no solo e, consequentemente, na análise da resposta da vegetação às condições do ambiente de crescimento (Ghiberto et al., 2011; Martín-Vertedor et al., 2011; Zhang et al., 2010).

\section{Conteúdo de água e armazenamento de água no solo com clones de palma forrageira}

Na Figura 3, é mostrada a variabilidade temporal do conteúdo médio de água do perfil do solo, até $0,70 \mathrm{~m}$, cultivado com três clones de palma forrageira. Verificou-se a rápida resposta do $\theta$ aos eventos de precipitação pluviométrica, sobretudo depois dos períodos de longa estiagem (entre outubro e dezembro de 2010 e segundo semestre de 2011), uma vez que o armazenamento de água era baixo e, com isso, favorecia a capacidade de infiltração de água no solo. Os menores valores do $\theta$ foram da ordem de $0,0797 \pm 0,0106 \mathrm{~m}^{3} \mathrm{~m}^{-3}$, enquanto os maiores ocorreram no período mais chuvoso (janeiro a maio de 2011), quando atingiram valores máximos da ordem de $0,2630 \pm 0,01 \mathrm{~m}^{3} \mathrm{~m}^{-3}$. Esse valor esteve próximo ao è de saturação, que, em média, foi de $0,3179 \mathrm{~m}^{3} \mathrm{~m}^{-3}$.

Não houve diferença do $\theta$ entre as parcelas cultivadas com os três clones. A área cultivada com o clone IPA Sertânia apresentou $\theta$ médio de 0,1782 $\pm 0,0420 \mathrm{~m}^{3} \mathrm{~m}^{-3}$,

Tabela 2. Parâmetros da equação de ajuste da condutividade hidráulica $\left(K(\theta), \mathrm{m} \mathrm{h}^{-1}\right)$ e da equação exponencial de ajuste do potencial total de água no solo $\left(\psi \mathrm{t}, \mathrm{m} \mathrm{m}^{-1}\right)$ ambos em função do conteúdo de água no solo $\left(\mathrm{m}^{3} \mathrm{~m}^{-3}\right)$, ao longo do perfil, obtidos pelo método do perfil instantâneo em um Argissolo Vermelho-Amarelo, em Serra Talhada, Semiárido pernambucano

\begin{tabular}{|c|c|c|c|c|c|c|c|}
\hline \multirow{2}{*}{ Camada } & \multicolumn{4}{|c|}{$\mathbf{K}(\theta)=\operatorname{Ko.exp}[\gamma \cdot(\theta-o)]$} & \multicolumn{3}{|c|}{$\psi t=-\alpha . \exp (-\beta . \theta)$} \\
\hline & $\operatorname{Ko}\left(\mathbf{m ~ h}^{-1}\right)$ & $\gamma\left(\mathbf{m}^{-1}\right)$ & $\theta o\left(\mathbf{m}^{3} \mathrm{~m}^{-3}\right)$ & $\mathbf{R}^{2}$ & $\alpha$ & $\beta$ & $\mathbf{R}^{2}$ \\
\hline $20 \mathrm{~cm}$ & 0,001 & 30,2163 & 0,2987 & 0,9951 & 34,2089 & 13,3638 & 0,9807 \\
\hline $30 \mathrm{~cm}$ & 0,0006 & 28,4565 & 0,3046 & 0,9913 & 26,4185 & 12,3038 & 0,9871 \\
\hline $40 \mathrm{~cm}$ & 0,0004 & 37,6967 & 0,3007 & 0,9843 & 52,048 & 14,8295 & 0,9772 \\
\hline $50 \mathrm{~cm}$ & 0,0002 & 57,8477 & 0,2940 & 0,9759 & 249,9288 & 20,2236 & 0,9575 \\
\hline $60 \mathrm{~cm}$ & 0,0001 & 67,8947 & 0,3105 & 0,9650 & 204,8216 & 17,8111 & 0,9301 \\
\hline $70 \mathrm{~cm}$ & 0,0001 & 77,333 & 0,3557 & 0,9674 & 789,0253 & 19,151 & 0,9378 \\
\hline $80 \mathrm{~cm}$ & 0,00007 & 95,7574 & 0,3609 & 0,9619 & 3338,61060 & 22,4891 & 0,9159 \\
\hline Média & 0,0004 & 56,4575 & 0,3179 & - & 670,7231 & 17,1674 & - \\
\hline $\mathrm{CV}$ & 75,94 & 36,94 & 7,27 & - & 118,69 & 18,32 & - \\
\hline
\end{tabular}

Ko = condutividade de água no solo no tempo de redistribuição igual a zero, $\gamma=$ parâmetro de ajuste da equação; $\mathrm{R}^{2}=$ coeficiente de determinação ajustado; $\alpha$ e $\beta$ = parâmetros de ajuste da equação $(n=49)$. 
enquanto para a Orelha de Elefante Mexicana foi de $0,1727 \pm 0,0341 \mathrm{~m}^{3} \mathrm{~m}^{-3}$ e para de Miúda foi de $0,1655 \pm$ $0,0453 \mathrm{~m}^{3} \mathrm{~m}^{-3}$.

Pela Figura 3B, constata-se que houve uma maior extração de água, até os $0,30 \mathrm{~m}$ de profundidade, pela Orelha de Elefante Mexicana, reduzindo-se progressivamente com o aumento de profundidade do solo. Até os $0,30 \mathrm{~m}$ de profundidade, não se observaram diferenças na extração de água pela IPA Sertânia e Miúda. Entretanto, para esse último clone, a partir dessa profundidade há uma ligeira redução do $\theta$. Um pico de extração é observado aos 0,50 $\mathrm{m}$ de profundidade, tendência essa semelhante à verificada para a IPA Sertânia, porém com magnitudes menores, logo que a sua extração de água reduz-se drasticamente a partir dos $0,30 \mathrm{~m}$, em relação aos dois outros clones.

Esse comportamento pode estar associado ao gênero Nopalea sp., especificamente quanto ao crescimento do sistema radicular, que pode ser diferente daquele do gênero Opuntia sp. Entretanto, esse resultado também pode variar entre espécies do mesmo gênero (Snyman, 2004), pois se percebe que, nas maiores profundidades, apesar de a Miúda apresentar extração menor nas primeiras camadas, naquelas mais profundas, a sua capacidade de extração tende a se assemelhar à da Orelha de Elefante Mexicana e é maior que a da IPA Sertânia.
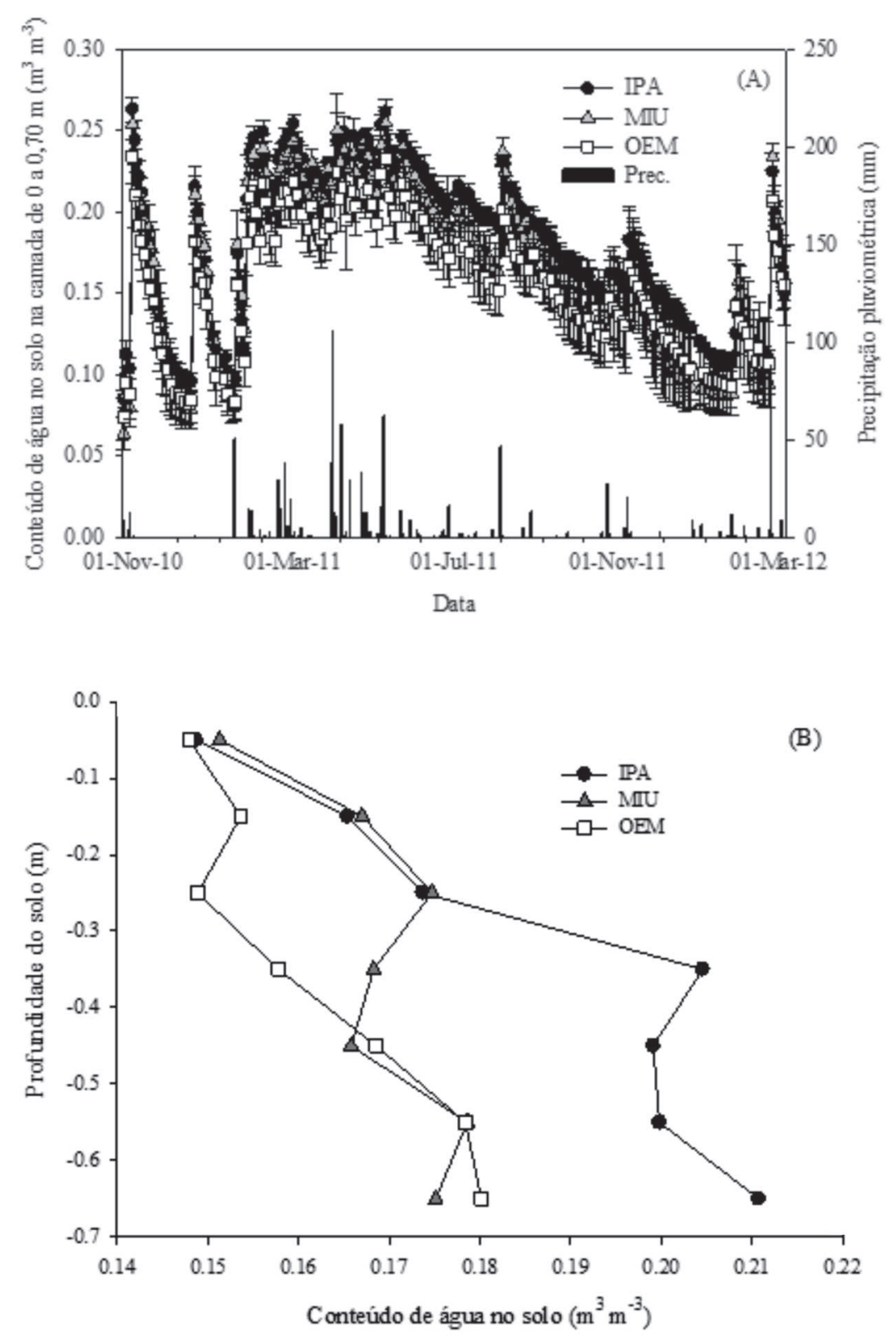

Figura 3. Variação temporal (A) e espacial (B) do conteúdo de água de um Argissolo cultivado com três clones de palma forrageira (IPA Sertânia - Nopalea sp., Miúda - Nopalea sp., Orelha de Elefante Mexicana - Opuntia sp.), em sequeiro, nas condições edafoclimáticas do Semiárido pernambucano. 


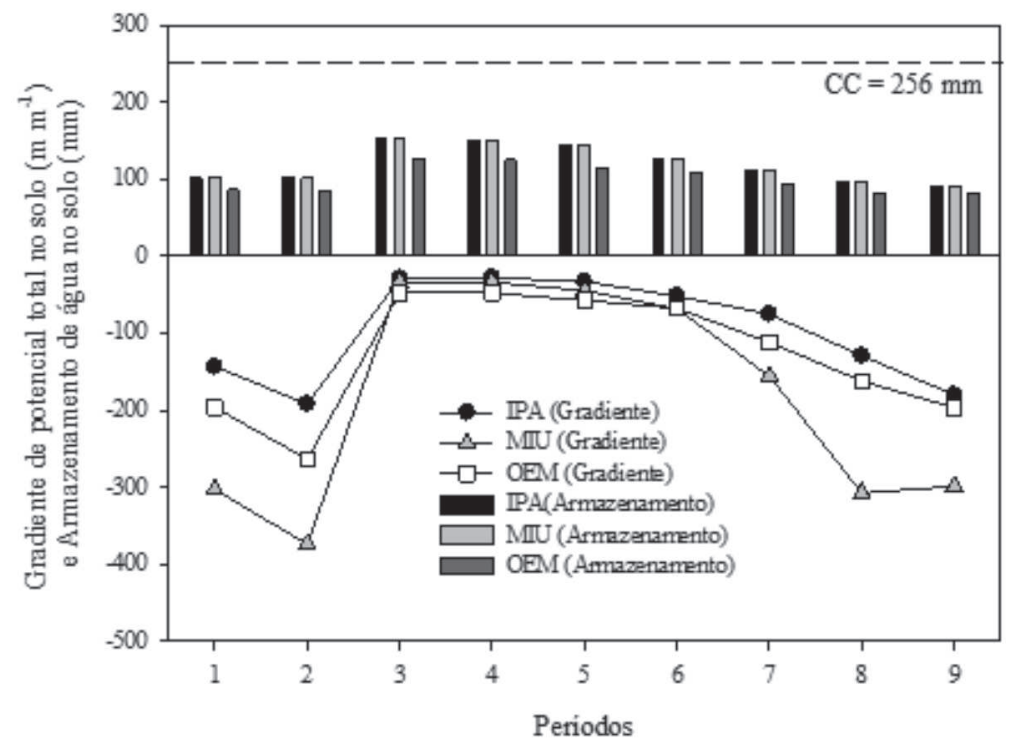

Figura 4. Gradiente do potencial total de água e armazenamento de água para uma profundidade de até 0,70 $\mathrm{m}$ de um Argissolo Vermelho-Amarelo cultivado com três clones de palma forrageira (IPA Sertânia - Nopalea sp., Miúda - Nopalea sp., Orelha de Elefante Mexicana - Opuntia sp.) nas condições edafoclimáticas do Semiárido Nordestino, totalizando 499 dias, representados em nove períodos.

Apesar de não se apresentarem diferenças significativas nos valores de $\theta$, verificaram-se, ao longo do tempo, distintas tendências na dinâmica de água no solo, nas áreas cultivadas com os três clones de palma forrageira, conforme Figura 4. Nessa mesma figura, é mostrado o potencial total médio de água do solo para a profundidade de $0,70 \mathrm{~m}(\Delta \psi \mathrm{t} / \Delta \mathrm{z})_{0,70 \mathrm{~m}}$ e o armazenamento de água no solo (A) desde 20 de outubro de 2010 até 02 de março de 2012, 499 dias, divididos em nove períodos representativos. $\mathrm{O}(\Delta \psi \mathrm{t} / \Delta \mathrm{z})_{0,70 \mathrm{~m}}$ é baixo durante os períodos em que se tem o menor valores de A, mas percebe-se que, para as áreas com cultivo da Miúda, é ainda mais reduzido, indicando que o fluxo descendente de água é menos favorecido, quando comparado com o das áreas dos outros dois clones, logo que se tem menor quantidade de água no perfil. Os menores gradientes foram verificados nos dois primeiros períodos ( 1 e 2 ) e nos três últimos ( 7,8 e 9), quando ocorreram as maiores estiagens. Em todos eles, o A não atingiu a capacidade de campo (CC), sendo sempre menor do que $60 \%$ da CC. Entretanto, quando A foi maior (períodos de 3 a 6), graças à ocorrência dos maiores volumes de precipitação, observou-se que o $(\Delta \psi t$ t $\Delta \mathrm{z})_{0,60 \mathrm{~m}}$ atingiu valores superiores $\left(>100 \mathrm{~m} \mathrm{~m}^{-1}\right.$ ), o que proporcionou o fluxo descendente, ou seja, a drenagem profunda, inibindo que os valores de A atinjam a capacidade de campo. Tendências similares foram mencionadas por Ghiberto et al. (2011). Os valores de A apresentaram magnitudes menores, nas áreas de cultivo com a Orelha de Elefante Mexicana, e maiores, nas áreas com a IPA Sertânia, confirmando a maior capaci- dade de extração de água do solo pelo primeiro clone. Essas distintas tendências dos valores de $\mathrm{A}$, ao longo do tempo, não revelaram diferenças significativas entre os clones.

\section{CONCLUSÕES}

As sondas capacitivas (modelo Diviner@2000) apresentaram bons desempenhos na estimativa do conteúdo de água do solo por meio de medidas de frequência relativa, em um Argissolo Vermelho-Amarelo, do Semiárido pernambucano, quando se comparam seus resultados com os relatados na literatura.

As equações físico-hídricas apresentaram ótimos ajustes na estimativa da condutividade hidráulica do solo e do potencial total de água no solo, em função do conteúdo de água do solo.

O conteúdo e o armazenamento de água no solo não variaram significativamente, mesmo quando cultivado com clones de palma forrageira de gêneros diferentes.

\section{REFERÊNCIAS}

Andrade Júnior AS, Silva CR \& Daniel R (2007) Calibração de um sensor capacitivo de umidade em Latossolo Amarelo na microrregião do Litoral Piauiense. Agrária, 2:303-307.

Carvalho LA de, Libardi PL, Rocha GC \& Cruz ACR (2007) Caracterização hidráulica de um latossolo vermelho associado à caracterização pedológica do perfil. Ciência Rural, 37:1008-1013.

Dourado-Neto D, Nielsen DR, Hopmans JW, Reichardt K \& Bacchi OOS (2000) Software to model soil water retention curves (SWRC, version 2.00). Scientia Agricola, 57:191-192. 
Dubeux Júnior JCB, Santos MVF dos, Lira M de A, Santos DC dos, Farias I, Lima LE \& Ferreira RLC (2006) Productivity of Opuntia fícus indica (L.) Miller under different $\mathrm{N}$ and $\mathrm{P}$ fertilization and plant population in northeast Brazil. Journal of Arid Enviroments, 67:357372.

EMBRAPA - Empresa Brasileira de Pesquisa Agropecuária (1999) Sistema Brasileiro de Classificação de Solos. Brasília, EMBRAPA/SPI. 412p.

Fares A, Buss P, Dalton M, El-Kadi AI \& Parsons LR (2004) Dual field calibration of capacitance and neutron soil water sensors in a shrinking-swelling clay soil. Valdose Zone Journal, 4:1390-1399.

Ghiberto PJ, Libardi PL, Brito AS \& Trivelin PCO (2011) Components of the water balance in soil with sugarcane crops. Agricultural Water Management, 102:1-7.

Gonçalves ADM de A (2011) Uma análise da determinação da condutividade hidráulica do solo pelo método do perfil instantâneo. Tese de Doutorado. Escola Superior de Agricultura "Luiz de Queiroz", Piracicaba. 123p.

Groves SJL \& Rose SCL (2004) Calibration equations for Diviner 2000 capacitance measurements of volumetric soil water content of six soils. Soil Use and Management, 20:96-97.

Hidalgo J, Pastor M \& Hidalgo JC (2003) Evaluacion de uma sonda FDR para la estimación del contenido de água en el suelo para el control de riegos en Olivar. Estudios de La Zona No Saturada del Suelo, 6:171-176

Jong Van Lier Q \& Libardi PL (1999) Variabilidade dos parâmetros da equação que relaciona a condutividade hidráulica com a umidade do solo no método do perfil instantâneo. Revista Brasileira de Ciência do Solo, 23:1005-1014

Libardi PL (2005) Dinâmica da água no solo. São Paulo, Editora da Universidade de São Paulo. 335p.

Martins MV (2009) Influência da distância de instalação de tensiômetros em campo para calculo do gradiente de potencial total no método do perfil instantâneo. Dissertação de Mestrado. Escola Superior de Agricultura “Luiz de Queiroz", Piracicaba. 77p.

Martín-Vertedor AI, Rodríguez JMP, Losada HP \& Castiel EF (2011) Interactive responses to water deficits and crop load in olive (Olea europaea L., cv. Morisca). II: Water use, fruit and oil yield. Agricultural Water Management, 98:950-958.
Oliveira FT de (2008) Crescimento do sistema radicular da Opuntia fícus-indica (L.) Mill (palma forrageira) em função de arranjos populacionais e adubação fosfatada. Dissertação de mestrado. Universidade Federal de Campina Grande, Patos. 76p.

Paltineanu IC \& Starr JL (1997) Real-time soil water dynamics using multisensor capacitance probes: laboratory calibration. Soil Science Society of America Journal, 61:1576-1585.

Prevedello BMS, Prevedello CL \& Libardi PL (1981) Simplificação analítica do método do perfil instantâneo para obtenção da condutividade hidráulica não saturada em condições de campo. Revista Brasileira de Ciência do Solo, 5:93-97.

Rossato L, Alvalá RC dos S \& Tomasella J (2004) Variação espaço-temporal da umidade do solo no Brasil: análise das condições médias para o período de 1971-1990. Revista Brasileira de Meteorologia, 19:113-122.

Santos TEM dos, Montenegro AAA \& Silva DD (2011) Umidade do solo no semiárido pernambucano usando-se reflectometria no domínio do tempo (TDR). Revista Brasileira de Engenharia Agrícola e Ambiental, 15:670-679.

Sentek (2000) Diviner 2000 user guide. Version 1.21. Stepney, Sentek Pty Ltd. 88p.

Silva AL da (2005) Variabilidade dos componentes do balanço hídrico: um estudo de caso em uma cultura do cafeeiro (Coffea arábica L.) no Brasil. Tese de Doutorado. Escola Superior de Agricultura "Luiz de Queiroz", Piracicaba. 72p.

Silva CR da, Andrade Júnior AS de \& Souza CF de (2008) Aspectos práticos na utilização da técnica de capacitância: desafios e aprendizagem. In: Souza CF (Ed.) Aplicações de Técnicas eletromagnéticas para o monitoramento ambiental. Taubaté, Unitau. p.25-45.

Silva JRL da, Montenegro AAA \& Santos TEM dos (2012) Caracterização física e hidráulica de solos em bacias experimentais do semiárido brasileiro, sob manejo conservacionista. Revista Brasileira de Engenharia Agrícola e Ambiental, 16:27-36.

Snyman HA (2004) Effect of various water application strategies on root development of Opuntia ficus-indica and O. robusta. J. PACD, 1:35-61.

Zhang B, Kang S, Li F, Tong L \& Du T (2010) Variation in vineyard evapotranspiration in an arid region of northwest China. Agricultural Water Management, 97:1898-1904. 